Pacific Journal of Mathematics

A GENERALIZATION OF THE PRIME RADICAL IN 


\title{
A GENERALIZATION OF THE PRIME RADICAL IN NONASSOCIATIVE RINGS
}

\author{
Hyo Chul Myung
}

In [5] Tsai defined the Brown-McCoy prime radical for Jordan rings in terms of the quadratic operation and proved basic results for the radical. In this paper we give a definition of the prime radical for arbitrary nonassociative rings in terms of a *-operation defined on the family of ideals and of a function $f$ of the ring into the family of ideals in the ring. The prime radical for Jordan or standard rings is obtained by a particular choice of the *-operation and the function $f$. We also extend the results for the Jordan case to weakly $W$ admissible rings which include the generalized standard rings and therefore alternative and standard rings as well as Jordan rings.

1. Let $K$ be any nonassociative ring and let $\mathscr{F}(K)$ denote the family of ideals of $K$.

Definition 1. We define a $*$-operation as a mapping of $\mathscr{J}(K) \times$ $\mathscr{I}(K)$ into the family of additive subgroups of $K$ such that

(*1) for $A, B, C$, and $D$ in $\mathscr{F}(K)$ if $A \subseteq C$ and $B \cong D$, then $A * B \subseteq C * D$,

$(* 2) \quad(0) * A=B *(0)=(0)$ for all $A, B$ in $\mathscr{J}(K)$,

(*3) $\quad \overline{A * B}=\bar{A} * \bar{B}$ for any homomorphic images $\bar{A}$ and $\bar{B}$ of $A$ and $B$ in $\mathscr{F}(K)$.

If $K$ is a Jordan ring, let $U_{x} \equiv 2 R_{x}^{2}-R_{x^{2}}$ be the quadratic operation and $A U_{B}$ be the additive subgroup of $K$ generated by $x U_{y}$, $x \in A$ and $y \in B$. Then the $U$-operation satisfies the conditions above. If the characteristic is not 2 , it is shown in [5] that $A U_{A}=A A^{2}$ and is an ideal of $K$ for $A$ in $\mathscr{J}(K)$.

For any ring $K$ and $A, B$ in $\mathscr{J}(K)$, if we define $A * B$ as the additive subgroup $A B^{2}+B^{2} A+(A B) B+(B A) B$, then $A * B$ also satisfies the conditions in Definition 1 . In case $K$ is a standard ring, it is shown in [6] that $A * B$ is an ideal of $K$ for $A, B$ in $\mathscr{J}(K)$. If $K$ is commutative or anticommutative, then $A * B=A B^{2}+(A B) B$. In particular, if $K$ is a Lie ring, $A * B$ is an ideal of $K$. Since $A^{2}$ is not in general an ideal of $K$ for $A$ in $\mathscr{J}(K)$, but there are considerably broad classes of nonassociative rings in which $A^{3} \equiv A A^{2}+A^{2} A$ is an ideal of $K$ for every ideal $A$, this example will be particularly interesting.

We recall that a noncommutative Jordan ring $K$ is one satisfying 
the flexible law $(x, y, x)=0$ and the Jordan identity $\left(x, y, x^{2}\right)=0$ for all $x, y$ in $K$, where $(x, y, z)=(x y) z-x(y z)$. Most of the well known nonassociative rings are included in the class of noncommutative Jordan rings. Recently Thedy [4] defined a considerably broad class of algebras that generalizes many of the well known algebras.

Definition 2. A noncommutative Jordan ring $K$ is called weakly $W$-admissible if it satisfies

$$
[(a, b, c), c]-([a, c], c, b)=0,
$$

and

$$
\begin{aligned}
& ([a, b], d, c]+([b, c], d, a)+([c, a], d, b] \\
= & p[(a, b, c), d]+q[S(a, b, c), d]+r[d,[b,[a, c]]]
\end{aligned}
$$

for some integers $p, q, r$ such that either $m(p, q, r) \equiv 3+2 p+6 q-$ $4 r \neq 0$, or $n(p, r) \equiv p+4 r \neq 0$, where $[a, b]=a b-b a$ and $S(a, b, c)=$ $(a, b, c)+(b, c, a)+(c, a, b)$.

Thedy called a noncommutative Jordan algebra over a field $W$-admissible if it satisfies the identity $[a,(a, a, b)]=0$ and the two identities above for $p, q, r$ in the field such that either $m(p, q, r) \neq 0$ or $n(p, r) \neq$ 0 . He proved that if the characteristic is not 2 , then any generalized standard ring of Schafer [2] is $W$-admissible with $p=-2$ and $q=r=$ 0. Therefore, weakly $W$-admissible rings include generalized standard rings and hence alternative and standard rings as well as Jordan rings. In case the characteristic is not 2 , it is also shown in [4, p. 192] that in any weakly $W$-admissible ring $K, A^{3}$ is an ideal of $K$ for $A$ in $\mathscr{J}(K)$.

Lemma 1.1. Let $K$ be any ring. Then the conditions $(* 2)$ and (*3) imply

(i) $(A+C) *(B+C) \subseteq A * B+C$, and

(ii) $A * B \subseteq A \cap B$ for ideals $A, B, C$ of $K$.

Proof. Consider the quotient ring $\bar{K}=K / C$, then by $(* 3)(\overline{A+}$ $\overline{C) *(B+C)}=\bar{A} * \bar{B}=\overline{A * B}$, and hence (i). Let $\bar{K}=K / A$, then $\overline{A * B}=$ $\bar{A} * \bar{B}=(\overline{0}) * \bar{B}=(\overline{0})$ by $(* 2)$ and so $A * B \subseteq A$. Similarly $A * B \subseteq B$ and $A * B \subseteq A \cap B$.

Definition 3. Let $K$ be any ring. Then $f$ is defined as a function of $K$ into $\mathscr{F}(K)$ such that for every $a$ in $K$

(f 1) $a \in f(a)$,

(f 2) if $x \in f(\alpha)$, then $f(x) \subseteq f(\alpha)$, 
(f 3) $\overline{f(a)}=f(\bar{a})$, where $\bar{a}$ is a homomorphic image of $a$.

The principal ideal (a) generated by $a$ in $K$ is an example of $f(a)$. Now let $S$ be a subset of $K$ and define $f(a)$ to be the ideal $(a, S)$ generated by $a$ and $S$. Then $f$ satisfies the conditions above. A similar function to $f$ has been defined in [1] for the associative case and in [3].

Henceforth we assume that $f$ denotes a function of $K$ into $\mathscr{I}(K)$ satisfying ( $\mathrm{f} 1$ ), (f 2 ), and (f 3 ). Then clearly $(\alpha) \subseteq f(\alpha)$. For an ideal $A$ of $K$, we denote the ideal $\sum_{a \in A} f(a)$ by $f(A)$. Then $A \subseteq f(A)$ and $f(A) \subseteq f(B)$ if $A \subseteq B$, and also $f((a))=f(a)$. But in general $f(A) \neq A$ as shown by the example $f(a)=(a, S)$ for a subset $S$ of $K$. Let $\mathscr{J}^{\prime}(K)$ denote the family of ideals $f(A)$ for $A$ in $\mathscr{J}(K)$. Then $\mathscr{J}^{\prime}(K) \cong$ $\mathscr{J}(K)$ and in particular, if $f$ is such that $f(a)=(a)$ for all $a$ in $K$, then $f(A)=A$ and $\mathscr{I}^{\prime}(K)=\mathscr{I}(K)$.

2. In this section we give a definition of the prime radical for any ring in terms of the $*$-operation and the function $f$.

Lemma 2.1. Let $K$ be any ring where the *-operation and the function $f$ are defined. For an ideal $P$ of $K$, the following are equivalent:

(i) If $f(A) * f(B) \subseteq P$ for $A, B$ in $\mathscr{J}(K)$, then either $f(A) \subseteq P$ or $f(B) \subseteq P$.

(ii) If we have $f(A) \cap c(P) \neq \varnothing$ and $f(B) \cap c(P) \neq \varnothing$, then $f(A) * f(B) \cap c(P) \neq \varnothing$.

(iii) If $a$ and $b$ are in $c(P)$, then $f(a) * f(b) \cap c(P) \neq \varnothing$.

Proof. We need only to show that (ii) and (iii) are equivalent. Let $a$ and $b$ be in $c(P)$, then $f(a) \cap c(P) \neq \varnothing$ and $f(b) \cap$ $c(P) \neq \varnothing$. Hence (ii) implies (iii). Now let $A$ and $B$ be ideals of $K$ with $f(A) \cap c(P) \neq \varnothing$ and $f(B) \cap c(P) \neq \varnothing$. Let $a \in f(A) \cap c(P)$ and $b \in f(B) \cap c(P)$. Assuming (iii), we get $f(a) * f(b) \cap c(P) \neq \varnothing$ and by $(* 1) f(A) * f(B) \cap(P) \neq \varnothing$, thus (ii) holds.

Definition 4. (i) An ideal $P$ of $K$ is called $f^{*}$-prime if it satisfies any one of Lemma 2.1. A nonempty subset $M$ of $K$ is called an $f^{*}$ system if, for $A, B$ in $\mathscr{F}(K), f(A) \cap M \neq \varnothing$ and $f(B) \cap M \neq \varnothing$ imply $f(A) * f(B) \cap M \neq \varnothing$.

(ii) An ideal $P$ of $K$ is called $f^{*}$-semiprime if, for any ideal $A$ of $K, f(A) * f(A) \subseteq P$ implies $f(A) \subseteq P$. A nonempty subset $M$ of $K$ is called an $s f^{*}$-system if, for $A$ in $\mathscr{S}(K), f(A) \cap M \neq \varnothing$ implies $f(A) * f(A) \cap M \neq \varnothing$.

An ideal $P$ is $f^{*}$-prime if and only if $c(P)$ is an $f^{*}$-system. Similarly, an ideal $P$ is $f^{*}$-semiprime if and only if $c(P)$ is an $s f^{*}$ - 
system. Let $K$ be a Jordan or standard ring. If we define $A * B$ as $A U_{B}$ or as $A B^{2}+B^{2} A+(A B) B+(B A) B$ and define $f(a)$ as $(a)$ for every $a$ in $K$, then the defininition of $f^{*}$-prime and $f^{*}$-semiprime ideals coincide with those in [5] or in [6].

Definition 5. For $A$ in $\mathscr{J}(K), A^{*}=\left\{x \in K \mid\right.$ any $f^{*}$-system containing $x$ meets $A$ \} is called the $f^{*}$-radical of $A$. Similarly, $A_{*}=$ $\left\{y \in K \mid\right.$ any $s f^{*}$-system containing $y$ meets $\left.A\right\}$ is called the $s f^{*}$-radical of $A$.

Theorem 2.2. Let $A$ be an ideal of $K$. Then

(i) $A^{*}$ is the intersection of all the $f^{*}$-prime ideals $P_{i}$ containing A.

(ii) $A_{*}$ is the intersection of all $f^{*}$-semiprime ideals containing $A$.

(iii) $A_{*}$ is an $f^{*}$-semiprime ideal of $K$.

(iv) $A$ is $f^{*}$-semiprime if and only if $A=A_{*}$.

Proof. The proofs are essentially the same as in [5]. But to emphasize use of the $*$-operation and the function $f$ we prove only (i). Let $\bigcap_{i} P_{i}$ be the intersection of all the $f^{*}$-prime ideals $P_{i}$ of $K$ containing $A$. If $a \notin P_{i}$ for some $i$, then $a \in c\left(P_{i}\right)$, being an $f^{*}$-system, and $c\left(P_{i}\right) \cap A=\varnothing$. Hence $a \notin A^{*}$ and $A^{*} \subseteq \bigcap_{i} P_{i}$. Conversely, if $a \notin A^{*}$, then there exists an $f^{*}$-system $M$ with $a \in M$ but $A \cap M=\varnothing$. By Zorn's lemma we find a maximal ideal $P$ such that $P \supseteqq A$ but $P \cap M=\varnothing$. Let $B, C$ be ideals of $K$ such that $f(B) \cap c(P) \neq \varnothing$ and $f(C) \cap c(P) \neq \varnothing$. By the maximality of $P,(f(B)+P) \cap M \neq \varnothing$ and $(f(C)+P) \cap M \neq$ $\varnothing$. Since $M$ is an $f^{*}$-system, $\varnothing \neq(f(B)+P) *(f(C)+P) \cap M \leqq$ $(f(B) * f(C)+P) \cap M$ by Lemma 1.1 (i), thus $f(B) * f(C) \cap c(P) \neq \varnothing$. Hence $P$ is $f^{*}$-prime and $a \notin P$.

Lemma 2.3. Let $a$ be an element of $K$ and $S$ be an $s f^{*}$-system containing $a$. Then there exists an $f^{*}$-system $M$ such that $a \in M$ and $M \subseteq S$.

Proof. Let $a_{1}=a$, then $a_{1} \in f\left(a_{1}\right) \cap S$ and so $f\left(a_{1}\right) * f\left(a_{1}\right) \cap S \neq \varnothing$. Hence we obtain a set $M=\left\{a_{1}, a_{2}, \cdots, a_{n}, \cdots\right\}$ such that $a_{k+1} \in$ $f\left(a_{k}\right) \cap S$ and $M \subseteq S$. By Lemma 1.1 (ii) we note that $a_{k+1} \in f\left(a_{k}\right) * f\left(a_{k}\right) \subseteq$ $f\left(a_{k}\right)$ and so $f\left(a_{k+1}\right) \subseteq f\left(a_{k}\right)$. Let $p=\max (i, j)$, then $a_{p+1} \in f\left(a_{p}\right) * f\left(a_{p}\right) \cap$ $S \subseteq f\left(a_{i}\right) * f\left(a_{j}\right) \cap S$. Hence $f\left(a_{i}\right) * f\left(a_{j}\right) \cap M \neq \varnothing$ and $M$ is an $f^{*}$ system.

Therefore, as in [5], we have

Theorem 2.4. For any ideal $A$ of $K, A^{*}=A_{*} . A^{*}$ is called the $f^{*}$-prime radical of $A$. 
Definition 6. The $f^{*}$-prime radical, $R^{*}(K)$, of $K$ is the $f^{*}$ prime radical of the ideal (0). A ring $K$ is said to be $f^{*}$-semisimple if $R^{*}(K)=(0)$.

Lemma 2.5. Let $\bar{K}$ be a homomorphic image of $K$. If $M$ is an $f^{*}$-system of $K$, then so is $\bar{M}$ in $\bar{K}$.

Proof. Let $\bar{A}, \bar{B}$ be ideals of $\bar{K}$ such that $f(\bar{A}) \cap \bar{M} \neq \varnothing$ and $f(\bar{B}) \cap \bar{M} \neq \varnothing$, where $A$ and $B$ are ideals in $K$ containing the kernel. Recalling (f 3) and $A \subseteq f(A)$, these imply $f(A) \cap M \neq \varnothing$ and $f(B) \cap$ $M \neq \varnothing$. Since $M$ is an $f^{*}$-system, by (*3) and (f 3$)$ we see that $f(\bar{A}) * f(\bar{B}) \cap \bar{M} \neq \varnothing$.

Therefore, by Lemma 2.3 we easily see that any homomorphic image of an $f^{*}$-prime ideal containing the kernel is also $f^{*}$-prime. Hence we obtain

THeOREM 2.6. Let $K$ be a ring and $R^{*}(K)$ be the $f^{*}$-prime radical of $K$, then $R^{*}\left(K / R^{*}(K)\right)=(0)$, that is, $K / R^{*}(K)$ is $f^{*}$-semisimple.

Definition 7. A ring $K$ is called an $f^{*}$-prime ring if $(0)$ is an $f^{*}$-prime ideal in $K$.

Clearly, an $f^{*}$-prime ring is $f^{*}$-semisimple. Since any homomorphic image of an $f^{*}$-prime ideal is $f^{*}$-prime, if $P$ is an $f^{*}$-prime ideal in $K$ then $K / P$ is an $f^{*}$-prime ring. Let $\bar{K}=K / P$ be an $f^{*} A$ prime ring and let $f(A) * f(B) \subseteq P$, then $f(\bar{A}) * f(\bar{B}) \subseteq(\overline{0})$ and so $f(A) \subseteq P$ or $f(B) \subseteq$ $P$, thus $P$ is $f^{*}$-prime in $K$. Hence $P$ is an $f^{*}$-prime ideal of $K$ if and only if $K / P$ is an $f^{*}$-prime ring. Therefore, as for Jordan rings, we obtain

THEOREM 2.7. A ring $K$ is isomorphic to a subdirect sum of $f^{*-}$ prime rings if and only if $K$ is $f^{*}$-semisimple.

3. Throughout this section we assume that the $*$-operation satisfies the following additional condition:

(*4) $A * A=A^{3}$ and $A * A$ is an ideal of $K$ for $A$ in $\mathscr{F}(K)$.

We recall that if $K$ is a weakly $W$-admissible or Lie ring then $A * B=A B^{2}+B^{2} A+(A B) B+(B A) B$ satisfies $(* 4)$.

Theorem 3.1. Let $A$ be an ideal of a ring $K$ and $r \in A_{*}$. Then a power of $r$ belongs to $A$. Furthermore if $K$ is power-associative, then the $f^{*}$-radical $R^{*}(K)$ is a nil ideal in $K$.

Proof. Let $M$ be the multiplicatively closed system generated 
by $r$ in $K$. Then it follows from $(* 4)$ that $M$ is an $s f^{*}$-system containing $r$. Hence $M \cap A \neq \varnothing$. If $K$ is power-associative and $r \in$ $R^{*}(K)$, then $r^{k} \in(0)$ for some $k$ and so $R^{*}(K)$ is nil.

Therefore, the $f^{*}$-radical $R^{*}(K)$ is contained in the nil radical $N(K)$ (the maximal nil ideal in $K$ ).

Let $\mathscr{I}^{\prime}(K)$ denote the set of ideals $f(A)$ for $A$ in $\mathscr{J}(K)$. Then $\mathscr{J}^{\prime}(K) \cong \mathscr{I}(K)$.

THEOREM 3.2. A ring $K$ is $f^{*}$-semisimple if and only if $\mathscr{I}^{\prime}(K)$ contains no nonzero nilpotent ideal.

Proof. It follows from Theorem 2.2 (iv) that $K$ is $f^{*}$-semisimple if and only if the ideal $(0)$ is $f^{*}$-semiprime. If $f(A)$ is a nonzero nilpotent ideal for $A$ in $\mathscr{I}(K)$, there exist positive integers $u=3^{t}$ and $v=3^{t-1}$ such that $f(A)^{u}=(0)$ but $f(A)^{v} \neq(0)$. But then since $f(A)^{v} * f(A)^{v} \subseteq f(A)^{3 v}=f(A)^{u}=(0),(0)$ is not $f^{*}$-semiprime. Conversely, if $(0)$ is not $f^{*}$-semiprime, then there exists an ideal $f(A) \neq(0)$ such that $f(A) * f(A)=f(A)^{3}=(0)$, thus $f(A)$ is nilpotent.

CoROLLARY 3.3. The $f^{*}$-radical $R^{*}(K)$ contains all the nilpotent ideals in $\mathscr{F}^{\prime}(K)$.

Proof. Let $f(A)$ be a nilpotent ideal in $\mathscr{J}^{\prime}(K)$ and $\bar{K}=K / R^{*}(K)$, then $\overline{f(A)}=f(\bar{A}) \in \mathscr{F}^{\prime}(\bar{K})$, and $f(\bar{A})$ is nilpotent in $\bar{K}$. Since $\bar{K}$ is $f^{*}$-semisimple, by Theorem $3.2 f(\bar{A})=(\overline{0})$, thus $f(A) \subseteq R^{*}(K)$.

THEOREM 3.4. If $K$ is a ring and $\mathscr{I}^{\prime}(K)$ contains a maximal nilpotent ideal $S^{\prime}(K)$, then $R^{*}(K)=S^{\prime}(K)$.

Proof. By Corollary 3.3, $S^{\prime}(K) \leqq R^{*}(K)$. Let $\bar{K}=K / S^{\prime}(K)$, then $\mathscr{J}^{\prime}(\bar{K})$ contains no nonzero nilpotent ideal and by Theorem $3.2 R^{*}(\bar{K})=$ (0). If $r \notin S^{\prime}(K)$, then $\bar{r} \neq \overline{0}$ and so there exists an $f^{*}$-prime ideal $\bar{P}$ in $\bar{K}$ with $\bar{r} \notin \bar{P}$. From $(* 3)$ and (f 3 ) it follows that the inverse image $P$ of $\bar{P}$ is an $f^{*}$-prime ideal in $K$. But since $\bar{r} \notin \bar{P}, r \notin P$ and so $r \notin R^{*}(K)$, thus $R^{*}(K) \subseteq S^{\prime}(K)$.

Now suppose that $f(a)=(a)$ for every element $a$ in $K$. Then $\mathscr{F}(K)=\mathscr{I}^{\prime}(K)$. Hence by Theorem $3.2 K$ is $f^{*}$-semisimple if and only if $K$ has no nonzero nilpotent ideal, and $R^{*}(K)$ contains all nilpotent ideals of $K$. In this case the ideal $S^{\prime}(K)$ is a maximal nilpotent ideal $S(K)$ in $K$ and by Theorem $3.4 R^{*}(K)=S(K)$.

Let $K$ now be a finite dimensional $W$-admissible or Lie algebra over a field. Let $f(a)=(a)$ for all $a$ in $K$. If $K$ is $W$-admissible, then it is shown in [4] that the nil radical $N(K)$ is nilpotent and so the 
unique maximal nilpotent ideal $S(K)$. Hence by Theorem $3.4 R^{*}(K)=$ $N(K)=S(K)$. If $K$ is a Lie algebra, it is well known that $K$ has a maximal nilpotent ideal $S(K)$ and hence $R^{*}(K)=S(K)$.

\section{REFERENCES}

1. K. Murata, Y. Kurata and H. Marubayashi, A generalization of prime ideals in rings, Osaka J. Math., 6 (1969), 291-301.

2. R. D. Schafer, Generalized standard algebras, J. of Algebra, 12 (1969), 386-417.

3. M. F. Smiley, Application of a radical of Brown and McCoy to non-associative rings, Amer. J. Math., 72 (1950), 93-100.

4. A. Thedy, Zum Wedderburnschen Zerlegungssatz, Math. Z., 113 (1970), 173-195.

5. C. Tsai, The prime radical in a Jordan ring, Proc. Amer. Math. Soc., 19 (1968), 1171-1175.

6. L. J. Zettel, Radicals in standard rings, Ph. D. Thesis, Michigan State University, 1970.

Received March 19, 1971 and in revised form July 1, 1971.

UNIVERSITY OF NORTHERN IOWA 



\section{PACIFIC JOURNAL OF MATHEMATICS}

\section{EDITORS}

H. SAMELSON

Stanford University

Stanford, California 94305

C. R. HobBY

University of Washington

Seattle, Washington 98105
J. DUGUNDJI

Department of Mathematics

University of Southern California

Los Angeles, California 90007

RICHARD ARENS

University of California

Los Angeles, California 90024

\section{ASSOCIATE EDITORS}

E. F. BECKENBACH

B. H. NeumanN

F WoLF

K. YoshidA

\section{SUPPORTING INSTITUTIONS}

UNIVERSITY OF BRITISH COLUMBIA

UNIVERSITY OF SOUTHERN CALIFORNIA

CALIFORNIA INSTITUTE OF TECHNOLOGY

UNIVERSITY OF CALIFORNIA

MONTANA STATE UNIVERSITY

STANFORD UNIVERSITY

UNIVERSITY OF NEVADA

NEW MEXICO STATE UNIVERSITY

OREGON STATE UNIVERSITY

UNIVERSITY OF OREGON

OSAKA UNIVERSITY

UNIVERSITY OF TOKYO

UNIVERSITY OF UTAH

WASHINGTON STATE UNIVERSITY

UNIVERSITY OF WASHINGTON

$\stackrel{*}{*} \stackrel{*}{*} \stackrel{*}{*}{ }^{*}{ }^{*}$ MMEICAN MATHEMATICAL SOCIETY

NAVAL WEAPONS CENTER

Printed in Japan by International Academic Printing Co., Ltd., Tokyo, Japan 


\section{Pacific Journal of Mathematics}

\section{Vol. 42, No. $1 \quad$ January, 1972}

Tage Bai Andersen, On Banach space valued extensions from split faces ........

David Marion Arnold, A duality for quotient divisible abelian groups of finite

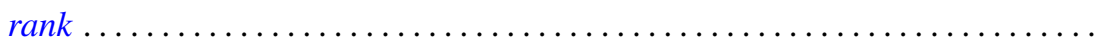

Donald Pollard Ballou, Shock sets for first order nonlinear hyperbolic

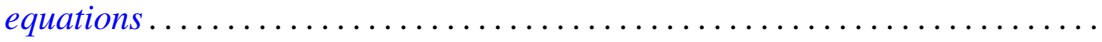

Leon Brown and Lowell J. Hansen, On the range sets of $H^{p}$ functions .........

Alexander Munro Davie and Arne Stray, Interpolation sets for analytic

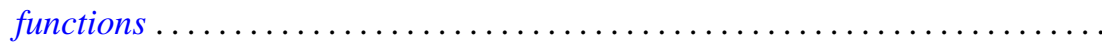

M. G. Deshpande, Structure of right subdirectly irreducible rings. II . . . . . . . . .

Barry J. Gardner, Some closure properties for torsion classes of abelian

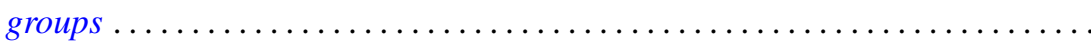

Paul Daniel Hill, Primary groups whose subgroups of smaller cardinality are

direct sums of cyclic groups . . . . . . . . . . . . . . . . . . .

Richard Allan Holzsager, When certain natural maps are equivalences .........

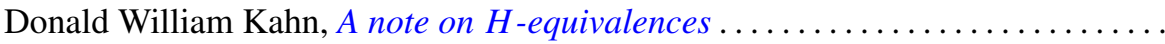

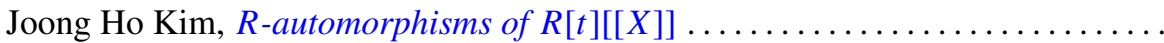

Shin'ichi Kinoshita, On elementary ideals of polyhedra in the 3-sphere.........

Andrew T. Kitchen, Watts cohomology and separability...

Vadim Komkov, A technique for the detection of oscillation of second order

ordinary differential equations .

Charles Philip Lanski and Susan Montgomery, Lie structure of prime rings of characteristic 2

Andrew Lenard, Some remarks on large Toeplitz determinants . .

Kathleen B. Levitz, A characterization of general Z.P.I.-rings. II .

Donald A. Lutz, On the reduction of rank of linear differential systems

David G. Mead, Determinantal ideals, identities, and the Wronskian ...

Arunava Mukherjea, A remark on Tonelli's theorem on integration in product

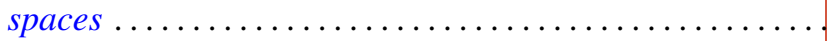

Hyo Chul Myung, A generalization of the prime radical in nonassociative rings.

John Piepenbrink, Rellich densities and an application to unconditionally nonoscillatory elliptic equations.

Michael J. Powers, Lefschetz fixed point theorems for a new class of multi-valued maps .

Aribindi Satyanarayan Rao, On the absolute matrix summability of a Fourier

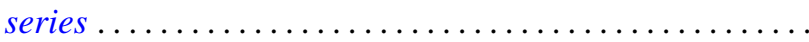

T. S. Ravisankar, On Malcev algebras ......................... 227

William Henry Ruckle, Topologies on sequences spaces . . . . . . . . . . . . . 235

Robert C. Shock, Polynomial rings over finite dimensional rings . . . . . . . . . 251

Richard Tangeman, Strong heredity in radical classes . . . . . . . . . . . . . . 259

B. R. Wenner, Finite-dimensional properties of infinite-dimensional spaces . . . . 267 